\title{
La imaginación creativa de estudiantes universitarios de la especialidad de Psicología
}

\author{
Lynn Katherine Zambrano Bravo ${ }^{1 a}$, Doris Elida Fuster Guillen ${ }^{2}$, Edgar Froilan Damian \\ Nuñez ${ }^{3}$, Miguel Gerardo Inga Arias $^{4}$ y Carmen del Pilar Gallardo Montes ${ }^{5}$ \\ Universidad César Vallejo, Lima, Perú ${ }^{1}$ \\ Universidad Nacional Mayor de San Marcos, Lima, Perú 234 \\ Universidad de Granada, Granada, España ${ }^{5}$ \\ Orcid ID: https://orcid.org/0000-0002-0561-9553 ${ }^{1}$ \\ Orcid ID: https://orcid.org/0000-0003-1319-4257² \\ Orcid ID: https://orcid.org/0000-0001-7499-84493 \\ Orcid ID: https://orcid.org/0000-0002-1588-01814 \\ Orcid ID: https://orcid.org/0000-0002-6510-2425
}

Recibido: 03 de febrero de 2018

Aceptado: 04 de noviembre de 2018

\section{Resumen}

El presente estudio tiene como finalidad determinar la dimensión predominante en la imaginación creativa de estudiantes de psicología, la imaginación creativa entendida como capacidad de inventar nuevas situaciones, productos o soluciones más allá de reproducirlas desde un conocimiento anterior. La investigación corresponde al tipo sustantivo, descriptivo explicativo, la muestra de estudio conformada por 85 estudiantes entre 16 a 18 años de la Facultad de Psicología de la Universidad César Vallejo, se utilizó un instrumento denominada PIC-J (Prueba de imaginación creativa para jóvenes), la validez de constructo se realizó a través del análisis de constructo y la confiabilidad a través del Alpha Cronbach. La contrastación de las hipótesis se realizó a través de la regresión logística y dichos resultados demuestran que la dimensión predominante es la creatividad narrativa y los indicadores relevantes la fluidez y originalidad, fundamental para los estudiantes.

Palabras claves: Imaginación creativa, fluidez, originalidad, creatividad narrativa.

\section{The creative imagination of university students of the specialty of Psychology}




\begin{abstract}
The purpose of this study is to determine the predominant dimension in the creative imagination of students of psychology, with creative imagination understood as the ability to invent new situations, products or solutions beyond reproducing them from previous knowledge. This research study design is substantive, descriptive and explanatory. The study sample is composed of 85 students between 16 and 18 years of the Faculty of Psychology of the César Vallejo University. An instrument called PIC-J (Test of creative imagination for young people) was used, construct validity was performed through construct analysis and reliability through the Cronbach Alpha. The testing of the hypotheses was made through the logistic regression and these results show that the predominant dimension is narrative creativity and the relevant indicators are fluency and originality, which are fundamental for the students.
\end{abstract}

Keywords: Creative imagination, fluidity, originality, narrative creativity.

\title{
Introducción
}

Internacionalmente, los cambios y la gran necesidad de la excelencia han ocasionado altas expectativas del profesional actual, teniendo casi la obligación de no solo formar sus conocimientos sino también de habilidades extras como la creatividad, tal como lo explica la Organización de las Naciones Unidas para la Educación, la Ciencia y la Cultura (UNESCO) en el 2008, donde menciona que la creatividad es un elemento esencial en el área económica de las personas y la sociedad. Esquivias (2004) aclaró que antiguamente el concepto de creatividad no era tan investigado y que solo hasta hace 40 años empezó a ser debidamente estudiado, significando ello el inicio de muchas investigaciones a nivel internacional que ponen a la creatividad en la relevancia necesaria como aspecto estrechamente relacionado al desarrollo integral de los individuos. El Perú no es un país ajeno a los avances y cambios que ocurren en el mundo, es así que Florida, Mellander y King (2015) en The Global Creativity Index muestra un ranking en el que nuestro país se ubica en el puesto 69 en cuanto a los niveles de creatividad en la población, evidenciando que el desarrollo de creatividad tiene aún un largo camino que recorrer a comparación de otros países latinoamericanos. En el Perú las investigaciones de la creatividad van avanzando de forma lenta pero segura, encontrando estudios recientes la importancia que tiene en el ámbito educativo, es así que referimos a 
Castillo (2007), quien menciona que el uso de recursos reciclables no solamente de aportar en el aprendizaje, también les permite desarrollar su creatividad. En el ámbito universitario existe alta demanda del desarrollo de la creatividad en los estudiantes puesto que la ausencia afecta competencia personal y profesional quienes requieren constante adaptación y rápida solución de problemas por parte de los docentes.

Cabe resaltar que existe una necesidad de estudiar dicha variable debido a que ayudará de forma significativa en diagnosticar, intervenir y enfatizar el trabajo en el desarrollo integral de los jóvenes, siendo la creatividad un elemento que le brindará posicionamiento y crecimiento a individuo en las diversas áreas de su vida, sobre todo en la etapa de su formación profesional que resulta ser el proceso de entrenamiento para su futuro desempeño laboral es por ello a realización del presente estudio.

\section{Imaginación creativa}

El inicio de nuestros conocimientos es la imaginación, se generan las ideas necesarias para luego proceder a la creación y la obtención de nuevos aprendizajes. (Barceló, 1989) Es así que, si se traslada esta concepción de la imaginación al ámbito académico, diríamos que resulta necesario esclarecer el nivel de imaginación, puesto que proporcionaría un panorama ideal para observar asimismo su nivel de creatividad y por ende su capacidad de adquirir y producir conocimientos.

Barceló (citado por González, 1998) mencionó que:

Nuestra libertad parte del poder imaginar, es lo que nos permite ser humanos más allá de todo lo racional. A la vez el ser imaginativo es parte de nuestra naturaleza, la cual origina la capacidad creativa tan necesaria para las diferentes áreas de nuestras vidas. (p. 132)

Es así que cuando se está interesado en determinados temas, tanto en su quehacer académico o personal, los individuos tienden a usar todas sus capacidades, entre ellas la creatividad. Asimismo, antes de involucrarse en cierta actividad el individuo creativo se sostiene en sus suposiciones y analizan las probabilidades que tienen de lograr modificar o reparar esa situación donde emplearán su pensamiento creativo. 
Morales (1998) señaló que la creatividad se relaciona con las actividades del cerebro que, además de almacenar y compartir vivencias anteriores, crea sobre todo nuevas ideas. La innovación es un pilar determinante en la capacidad creativa, en los estudiantes universitarios ello implicaría unificar todo lo vivenciado en la etapa escolar, es decir académicamente, y a la vez tomar lo aprendido a nivel personal para obtener un perfil de profesional capaz de lograr salidas innovadoras a sus dificultades o problemas cotidianos en su labor profesional. La base de la creatividad entonces, se oculta en el principio de todo, en las dudas y cuestionamientos que poseemos frente a las cosas y las situaciones, es a partir de ellas que surgen ideas y las elaboraciones más complejas. Si sólo nos ponemos como ejemplo las situaciones problemáticas a las que nos vemos inmersos a diario, todas esas dificultades parten del punto de no saber cómo accionar, de hacernos preguntas mentales inventando supuestos hasta llegar a la decisión final y posible solución

Arango y Henao (2006) explicaron lo siguiente:

La imaginación creativa resulta ser una especie de mezcla entre el factor intelectual y emocional, la cual origina la habilidad de utilizar visualizaciones y crear otras nuevas. Estimar esta área es importante hacer uso de la fantasía, del inventar. Es así que la imaginación creativa es un elemento sumamente valioso para hallar soluciones a las diversas situaciones problemáticas de nuestra vida diaria, incluso se encontró que el individuo creativo suele "ver" una solución del problema a modo de imagen, lo cual explicaría cómo los artistas expertos en pintura primero obtienen imágenes mentales antes de evocarlas en un lienzo y asimismo los escritores capaces de imaginar historias mentales antes de cada escritura. (p.4)

La imaginación creativa reflejaría entonces en los estudiantes la capacidad de inventar nuevas situaciones, productos o soluciones más allá de reproducirlas desde un conocimiento anterior. La imaginación creativa les brinda la oportunidad de sentir la "libertad" de alterar todo aquello que ya se sabe e introducirse en un contexto nuevo de muchas posibilidades. Herrera (2015) señaló que la creatividad y la imaginación son dos aspectos que van de la mano como componentes importantes de nuestra vida y que a la vez tienen un gran impacto en nuestras conductas. (p. 8) Dependerá mucho entonces, del nivel de creatividad e imaginación en los jóvenes que estos tengan determinado desenvolvimiento tanto en el área personal, académica o posteriormente en el área laboral.

En una aproximación al concepto de creatividad, Arango y Henao (2006) mencionaron acerca de la relación entre imaginación y creatividad y afirman que todo lo que 
nos rodea ha sido creado por el hombre en base a la imaginación, excepto la naturaleza. (p. 3). En relación a ello, Herrera (2015) señaló que la creatividad y la imaginación son dos elementos muy relacionados como aspectos importantes de nuestras vidas y que a la vez tienen una gran influencia en nuestros comportamientos. (p. 8). Al profundizar más en esta temática se encontró que Artola, Ancillo, Barraca y Mosteiro (2008) afirman que la Imaginación creativa hace referencia a la habilidad de obtener, soñar e ir más allá de lo ya visto, incluso más allá de lo real (p. 14). Asimismo, mencionaron que la creatividad es una variable muy compleja que requiere de la existencia de aspectos personales, donde encontramos: la creatividad narrativa que comprende la fluidez, la flexibilidad y la originalidad; y la creatividad gráfica que comprende la originalidad, elaboración, título y detalles especiales.

El contexto y la creatividad son dos aspectos relacionados estrechamente pues el entorno presentaría los retos que logren motivar la creatividad, es decir, cuando el entorno tiene dificultades se presta a la generación de ideas creativas, significando un reto y un aliento del pensamiento creativo, con situaciones que abran la posibilidad de emprender con ideas creativas. Según Artola et. al. (2008) refirieron que la imaginación creativa se refiere a la capacidad de visualizar y armar imágenes en la mente, de pensarlas sin que nadie antes lo haya hecho, yendo más allá de la realidad. (p. 14) La imaginación creativa sería de esta manera, un constructo que aportaría a la creación de nuevas propuestas que antes no se han presentado o siquiera se han imaginado otros sujetos, elementos novedosos y situaciones innovadoras frente a problemáticas, los autores afirmaron que la imaginación es lo que la gente menciona como soñar con los ojos abiertos, de hacer uso de la fantasía que se produce a nivel mental, a la capacidad de inventar, es decir, resulta ser un elemento sumamente importante y valioso que actúa como pilar para dar soluciones exitosas a las diversas problemáticas en la que nos vemos inmersos en la cotidianidad. Incluso muchos sujetos refieren que son capaces de visualizar las soluciones antes de expresarlas o plasmarlas en la realidad, al igual que un artista inicialmente tiene en su mente lo que reproducirá en el lienzo.

\section{Estructura de la imaginación creatividad}

Artola et al. (2008) revela que la imaginación creativa posee una estructura bidimensional: la primera denominada creatividad narrativa o verbal, que se refiere a la 
valoración de la capacidad de imaginar del individuo cuando hace uso de las palabras o el lenguaje que se encarga de la fluidez, flexibilidad y originalidad y la segunda creatividad figurativa o gráfica cuyo objetivo es lograr la observación de la imaginación cuando el sujeto hace uso de dibujos o gráficos centradas en la identificación de la originalidad, elaboración, título y detalles especiales.

\section{Estrategias para trabajar la creatividad:}

Sternberg y Lubart (citados por Casillas, 1999, p. 6) propusieron una serie de estrategias para aumentar el nivel de creatividad en los salones de clase, haciendo posible visualizarla como una capacidad que todos pueden desarrollar y no como algo exclusivo de solo algunas personas.

La inteligencia encuentra su importancia puesto que aporta en la generación de ideas, reestructurar las problemáticas y a la vez analizar si funcionarían. En este recurso se encontraría la relación entre el área sintética, analítica y práctica del sujeto. La inteligencia resulta ser un elemento que promueve la observación de la información como la selección, el combinar, etc. ayuda a discriminar entre una idea buena y una idea mala, a la vez considera qué problemáticas resultan lo suficientemente interesantes para tomar el impulso de resolverlas, evaluar los recursos que se tienen y cómo se actuará. De esta manera la inteligencia aporta de manera racional en la diferenciación de las ideas que van surgiendo en el camino hacia el logro de la solución de un problema, los estudiantes suelen verse inmersos en su quehacer diario a diversas dificultades que deberán analizar cuidadosamente, y es donde la inteligencia actuará de pilar importante.

En cuanto al conocimiento, es importante que el sujeto cuente con conocimientos previos acerca del área donde desea resolver una problemática, que cuente con esa experiencia que prepare el camino hacia un resultado esperado, a la vez que, conociendo sobre el tema, se podría garantizar que lo que está creando es realmente novedoso evitando caer en un procedimiento ya antes realizado, generando nuevas propuestas que superen a las anteriores además de contar con un sustento teórico para sus propuestas. Los estudiantes universitarios tendrían la necesidad de investigar o estudiar sobre un tema si se requiere llegar a un resultado óptimo respecto a alguna dificultad, estudiar por ejemplo sobre los trastornos 
afectivos le ayudará a llegar a un diagnóstico más preciso de un futuro paciente, sucediendo lo contrario si no cuenta con esta información.

Los estilos de pensamiento son las maneras en que los sujetos deciden hacer uso de las habilidades que posee. Stenberg (1999) los define del siguiente modo:

Un estilo es una manera característica de pensar. No se refiere a una aptitud, sino a cómo utilizamos las aptitudes que tenemos. No tenemos un estilo, sino un perfil de estilos. Las personas pueden ser prácticamente idénticas en cuanto a sus aptitudes y aun así tener estilos muy distintos (p. 38).

En este sentido, se hallan tres estilos: el ejecutivo, el legislativo y el judicial. Los distintos estilos determinarían la forma de enfrentarse a una determinada problemática, existiendo enfoques que propiciarían más que otros el desarrollo de la creatividad. Es así que el estilo legislativo presenta más relación con el planteamiento de circunstancias, con la iniciativa y el reto de enfrentar las dificultades que no están finalizadas, con plantear métodos audaces o poco usuales; los otros estilos del pensamiento se encuentran más relacionados a temas con más incidencia. Los sujetos que no son tienen el estilo legislativo necesitan saber con exactitud qué deben hacer y cómo lo deben hacer, prefieren evitar las confusiones puesto que no son tolerantes a las instrucciones ambiguas y suelen respetar mucho las normas. El pensamiento legislativo se vincula en mayor grado con el desarrollo de la creatividad, ya que, en palabras de Stenberg (1999), a las personas con este tipo de pensamiento "les gusta hacer las cosas a su aire y prefieren decidir por sí mismas qué harán y cómo lo harán. [...] les gusta establecer sus propias reglas y prefieren los problemas que no estén estructurados o planteados de antemano" (p. 49).

A la vez existen otros estilos de pensamiento estudiados por Sternberg y Lubart (1997) relacionados con la manera de organización de las ideas por parte de los sujetos: estilo monárquico, el jerárquico, el oligárquico y el anárquico. Estas formas se presentan como las más comunes. En el caso de la creatividad el estilo monárquico suele presentarse más frecuentemente en personas con altos niveles de creatividad como Picasso, Freud y Einstein; caracterizados por la energía con la que se manejaban en su quehacer, teniendo preferencia por finalizar una actividad antes de intentar dar inicio a otra, evidentemente preferían dedicarse de lleno a un solo proyecto a la vez. 
La personalidad actúa como otro elemento importante en la producción y hace referencia a cómo el sujeto se relaciona con su contexto, a cómo persevera frente a las dificultades y a su ánimo para asumir retos, su motivación por mejorar, su nivel de tolerancia a la diversidad de ideas, su apertura a las vivencias nuevas y también a su nivel de autoestima o confianza. Se demostró que involucrar todos estos aspectos en la formación de los estudiantes y su fortalecimiento se propicia un ambiente ideal para desarrollar la creatividad.

La motivación resulta de gran importancia puesto que actuaría como generador de la energía necesaria para explorar en los trabajos, de lo contrario, el proceso de búsqueda resultaría agotador. Los estudiantes en el avance de sus estudios y logros académicos suelen tener grandes motivaciones de diferentes tipos, los cuales mantienen en pie su actitud perseverante de continuar su camino profesional a pesar de las adversidades y problemas. De esta forma, un nivel alto de motivación estaría generando una sensación de bienestar no solo en la ejecución de la actividad sino también hacia los objetivos. De esta forma, como cita Polanco (2005), “cuando un estudiante tiene una motivación intrínseca, está motivado por la vivencia del proceso, más que por los logros o resultados del mismo, lo que provoca que estudie por el interés que le genera la materia” (p. 4). Los sujetos creativos por ello muestran un gran interés por lo que realizan puesto que su motivación suele ser intrínseca, logrando superar muchos obstáculos. En este aspecto, la motivación extrínseca no tendría un peso significativo a la creatividad, pero a pesar de ello podría ser relativo pues hay estudios recientes que muestran que las motivaciones externas también tendrían impacto en la producción creativa de los sujetos, sin embargo, no lograrían superar a la motivación intrínseca. En esta línea, resultan interesantes las palabras de Mas y Medinas (2007):

El profesorado universitario debe ser consciente de su responsabilidad como elemento motivador para el aprendizaje y poner en marcha todas las estrategias disponibles para fomentar la motivación intrínseca de sus alumnos, la orientación hacia el logro y el aumento de las expectativas de su alumnado. (p. 23)

\section{Metodología}

Se trabajó bajo el diseño no experimental, Hernández, Fernández y Baptista (2014) puesto que no se manipuló la variable y se centró en la observación de los fenómenos tal y como se hallan naturalmente, para luego analizarlos. (p. 149). El tipo de investigación sustantiva, estando la muestra constituida por 85 estudiantes de 16 a 18 años que se cursan 
el I ciclo de la carrera de Psicología. El instrumento utilizado fue la prueba de imaginación creativa para Jóvenes (PIC-J). La finalidad de la prueba es la evaluación de la creatividad narrativa y gráfica. La validez de constructo se realizó a través del análisis confirmatorio del ítem. En relación con la confiabilidad, se presenta un alfa de Cronbach de 0.942, lo que refleja una alta fiabilidad y coherencia interna.

\section{Resultados}

\section{Resultado general}

La dimensión predominante de la Imaginación creativa en estudiantes de psicología de I Ciclo menores de edad de una universidad privada de Lima Norte - 2017, es la creatividad gráfica.

Tabla 3

Coeficientes de la regresión logística de las dimensiones de la Imaginación creativa en estudiantes de Psicología de I Ciclo menores de edad de una universidad privada de Lima Norte - 2017

\begin{tabular}{lcccccc}
\hline \multicolumn{1}{c}{ Indicador } & B & $\begin{array}{c}\text { Error } \\
\text { estándar }\end{array}$ & Sig. & Exp(B) & \multicolumn{2}{c}{ 95\% C.I. para EXP(B) } \\
\cline { 6 - 7 } $\begin{array}{l}\text { Creatividad } \\
\text { narrativa }\end{array}$ & 2.479 & 0.590 & 0.000 & 11.924 & 3.750 & 37.913 \\
$\begin{array}{l}\text { Creatividad gráfica } \\
0.293\end{array}$ & 0.303 & 0.333 & 1.340 & 0.741 & 2.425
\end{tabular}

Fuente: Base de datos

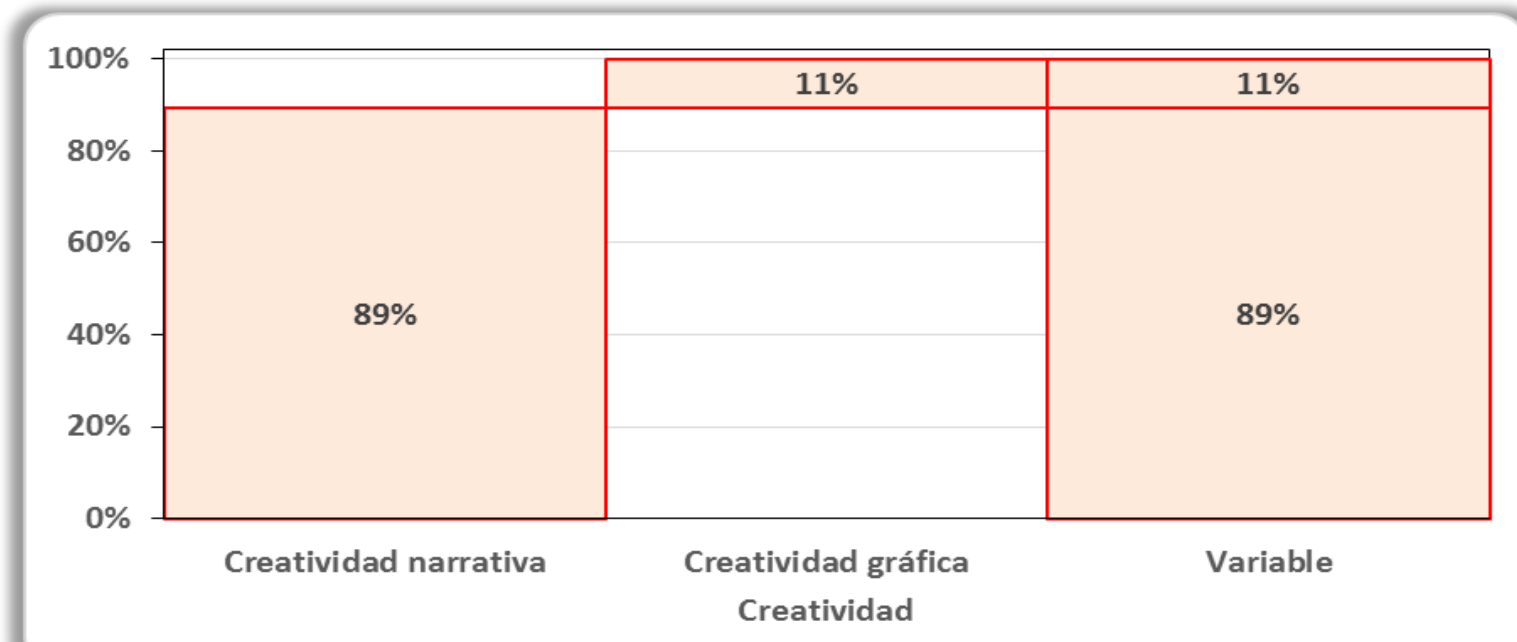


Figura 4. Pesos de las dimensiones de la Imaginación creativa en estudiantes de Psicología de I Ciclo menores de edad de una universidad privada de Lima Norte - 2017

Según los resultados, la dimensión creatividad narrativa $(B=2.479)$ tiene mayor coeficiente y logra aportar más en la imaginación creativa en los estudiantes del estudio. A la vez presenta un odds ratio $(\operatorname{Exp}(B))=11.924$, lo que significa que es una dimensión de riesgo, si un estudiante de psicología no desarrolla a creatividad narrativa podría presentar niveles bajos de imaginación creativa.

\section{Resultado específico 1}

El indicador que predomina en la Creatividad Narrativa en estudiantes de Psicología de I Ciclo de una universidad privada de Lima Norte - 2017 es la Fluidez.

Tabla 4

Coeficientes de la regresión logística de los indicadores de la dimensión creatividad narrativa en estudiantes de Psicología de I Ciclo menores de edad de una universidad privada de Lima Norte - 2017

\begin{tabular}{lcccccc}
\hline \multirow{1}{*}{ Indicador } & B & Error estándar & Sig. & Exp(B) & \multicolumn{2}{c}{$95 \%$ C.I. para EXP(B) } \\
\cline { 5 - 7 } & & & & & Inferior & Superior \\
\hline Fluidez & 2.705 & 1.083 & 0.012 & 14.960 & 1.792 & 124.864 \\
Flexibilidad & 0.549 & 0.797 & 0.492 & 1.731 & 0.363 & 8.259 \\
Originalidad & 1.034 & 0.563 & 0.066 & 2.812 & 0.933 & 8.473 \\
\hline
\end{tabular}

Fuente: Base 


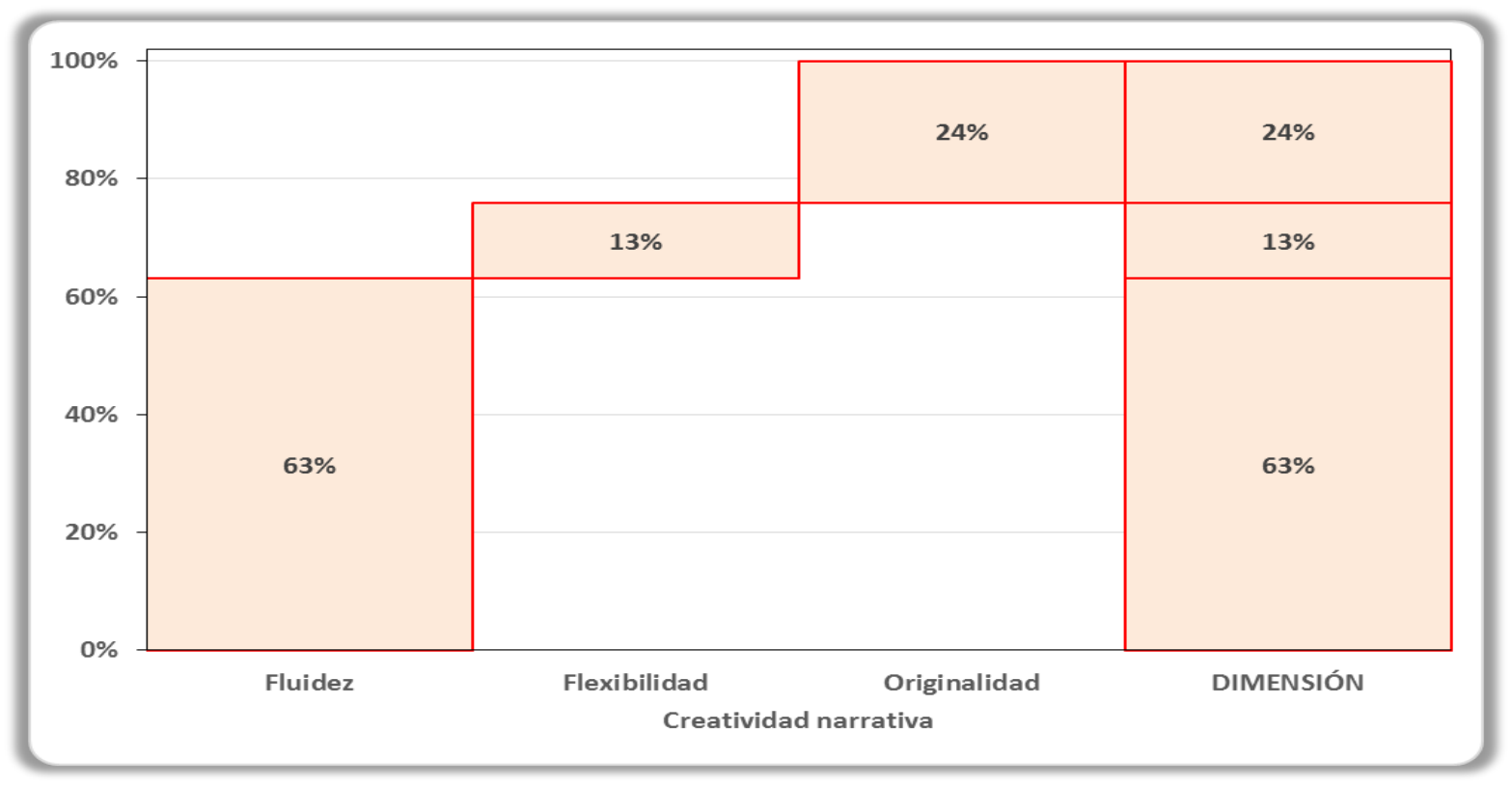

Figura 5. Pesos de los indicadores de la creatividad narrativa en estudiantes de Psicología de I Ciclo menores de edad de una universidad privada de Lima Norte - 2017

Según los resultados, el indicador fluidez $(B=2.705)$ tiene mayor coeficiente y por lo tanto aportaría más a la imaginación creativa narrativa en los participantes del estudio. A la vez esta dimensión presenta un odds ratio $(\operatorname{Exp}(B))=14.960$, lo cual significa que es un indicador de riesgo y que un alumno tiene 14.960 de veces de posibilidad de presentar niveles bajos de creatividad narrativa en relación a otro alumno con niveles altos de creatividad narrativa por causa del indicador fluidez. Por ello, se acepta la primera hipótesis específica de investigación, puesto que el indicador que predomina es la fluidez.

Resultado específico 2

El indicador que predomina en la Creatividad gráfica en estudiantes de Psicología de I Ciclo de una universidad privada de Lima Norte - 2017 es la originalidad.

\section{Tabla 5}

Coeficientes de la regresión logística de los indicadores de la dimensión creatividad gráfica en estudiantes de Psicología de I Ciclo menores de edad de una universidad privada de Lima Norte - 2017

\begin{tabular}{llllll}
\hline Indicador & B & $\begin{array}{c}\text { Error } \\
\text { estándar }\end{array}$ & Sig. & $\operatorname{Exp}(\mathrm{B})$ & \multicolumn{2}{c}{$95 \%$ C.I. para EXP(B) } \\
\cline { 5 - 6 } & & &
\end{tabular}




\begin{tabular}{lcccccc}
\hline Originalidad & 158.712 & 5663.900 & 0.978 & $8 \mathrm{E}+68$ & 0.000 & 0.000 \\
Elaboración & 64.644 & 2422.125 & 0.979 & $1 \mathrm{E}+28$ & 0.000 & 0.000 \\
Título & 102.216 & 3665.235 & 0.978 & $2 \mathrm{E}+44$ & 0.000 & 0.000 \\
Detalles especiales & 30.858 & 2033.595 & 0.988 & $3 \mathrm{E}+13$ & 0.000 & 0.000 \\
\hline
\end{tabular}

Fuente: Base de datos

El indicador de mayor peso es la originalidad, pero que ésta a su vez no es significativa estadísticamente. Por lo tanto, se rechaza la segunda hipótesis específica de investigación.

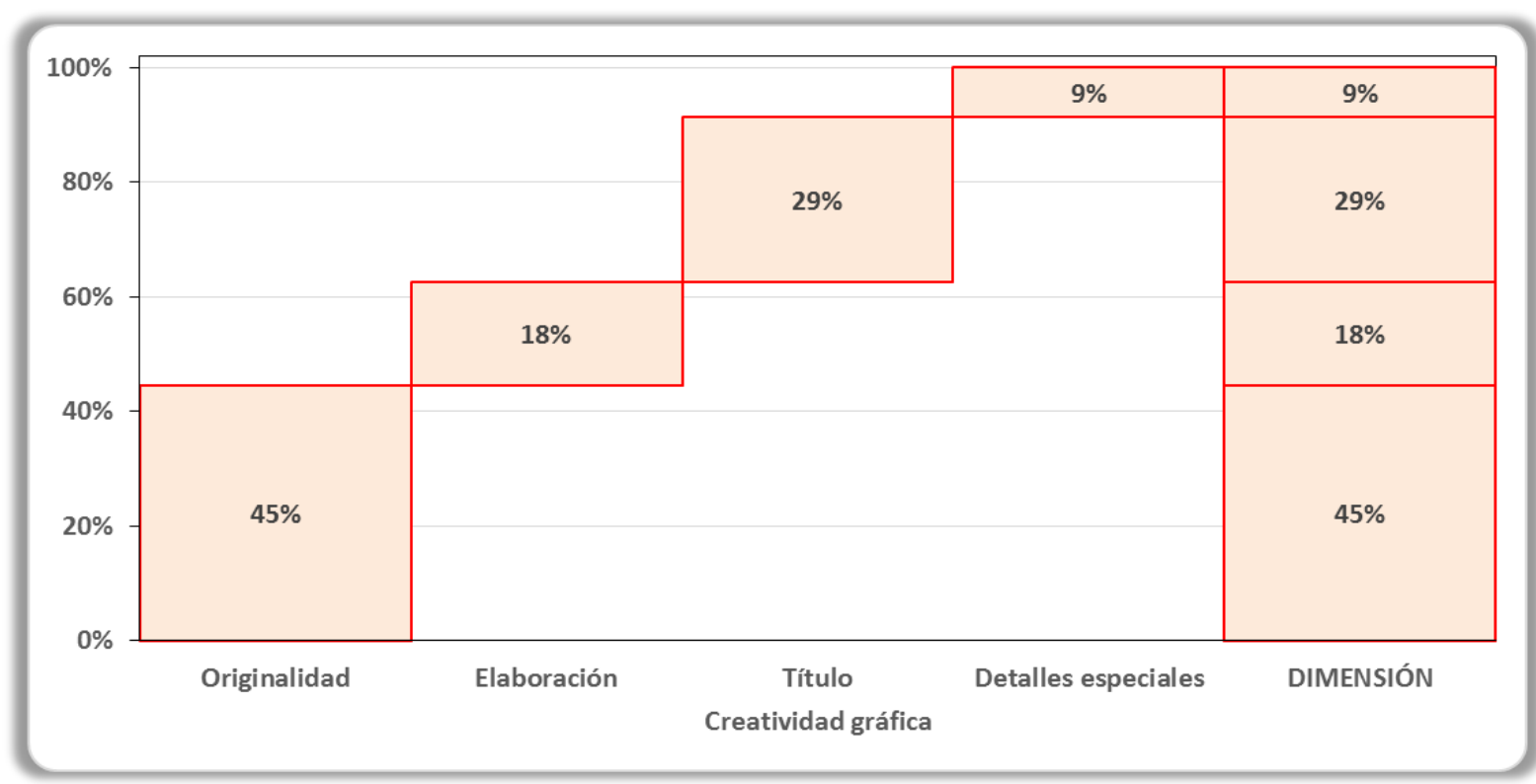

Figura 6. Pesos de los indicadores de la creatividad gráfica en estudiantes de Psicología de I Ciclo menores de edad de una universidad privada de Lima Norte - 2017

\section{Discusión}

En la presente investigación, se encontró que el 53\% de los estudiantes de Psicología de I Ciclo de una universidad de Lima presentan una regular imaginación creativa, mientras que el 7\% de los mismos presentan una buena imaginación creativa. Dicha acepción tiene relación con Ruschiensky (2015) en su investigación denominada "Competencia y creatividad en la traducción: Perspectivas Multilingües”, la cual concluyó en la importancia en el quehacer profesional y laboral en torno no solo a los conocimientos, sino también a la experiencia, las actitudes y la creatividad que aportan significativamente en el logro más efectivo de su labor. Se puede inferir que en el sistema educativo podría estar ocurriendo que se le otorga más peso en la formación académica a los conocimientos que pueda adquirir el estudiante en merma del desarrollo a nivel personal, de sus habilidades y sobre todo de la 
creatividad que actúa como un pilar importante hacia el logro de sus objetivos, por ello las cifras antes mencionadas se hallan inclinadas hacia el desarrollo regular de esta capacidad.

Asimismo, si profundizamos más en la variable encontramos que el $67 \%$ de los estudiantes de Psicología de I Ciclo de una universidad de Lima presentan una regular creatividad narrativa, mientras que el $7 \%$ de los mismos presentan una buena creatividad narrativa, resultado que está relacionado con lo obtenido por Cardona (2016) en la investigación denominada "El desarrollo de la creatividad y su relación con el rendimiento académico en las áreas de lenguaje, matemática y artística en estudiantes universitarios". Dicho estudio concluyó que, respecto a la prueba aplicada, la creatividad gráfica tiene más relación con asignaturas que denoten el desempeño artístico de los estudiantes, ocurriendo lo contrario con las asignaturas de lenguaje y matemática que tienen más relación con la creatividad narrativa y la creatividad en general. Se observa entonces que en la carrera de Psicología los estudiantes presentan una Creatividad narrativa ciertamente débil pero superior a la Creatividad gráfica, cuyo resultado se vería reafirmado con el estudio de Cardona (2016) puesto que la psicología tiene una fuerte relación con las actividades que denoten un buen desempeño en las áreas de lenguaje y matemáticas debido a las grandes cantidades de información y cálculos a los que desde el inicio de la carrera son sometidos los jóvenes.

A la vez se arribó al resultado que la dimensión creatividad narrativa $(B=2.479)$ presenta mayor coeficiente y por ende aporta más a la imaginación creativa en estudiantes de Psicología de I Ciclo menores de edad de una universidad privada de Lima Norte - 2017, resultado que se ve fortalecido con las conclusiones de Serna (2016) en el estudio “creatividad, inteligencias múltiples y estrategias de aprendizaje en maestras y maestros en formación" donde encontró relación entre creatividad y las inteligencias intra e interpersonal. De esta manera indicaría que la creatividad narrativa tendría un impacto en el desenvolvimiento de los jóvenes con su entorno mediante el uso de sus habilidades sociales y el lenguaje verbal y no verbal, teniendo facilidad de comunicarse, compartir y generar un intercambio de información necesario para desarrollar sus niveles de creatividad a la vez.

Dentro de la dimensión de Creatividad narrativa encontramos 3 indicadores: fluidez, flexibilidad y originalidad. Se ha obtenido que el indicador fluidez $(B=2.705)$ presenta mayor coeficiente y por ende aporta más a la imaginación creativa narrativa en estudiantes 
de Psicología de I Ciclo menores de edad de una universidad privada de Lima Norte - 2017, resultados que guardan relación con lo logrado por Reyes (2013) en la tesis "El debate dramatizado y el desarrollo de capacidades creativas de estudiantes en el curso taller de comunicación integral en la Facultad de Educación de la Universidad Nacional Mayor de San Marcos", concluyendo que la aplicación de técnicas que le permitan a los estudiantes la libertad de su expresión y uso de sus argumentos y opiniones propician un espacio de desarrollo para su creatividad. De esta forma quedaría demostrada la importancia de la participación de los alumnos en el aula en beneficio del incremento de sus niveles de creatividad, pudiendo luego ser aplicados en sus actividades como profesionales, logrando un mejor desempeño y significando también una ventaja para la eficacia de su labor. En tal sentido, se estaría demostrando que al promover un ambiente donde el estudiante pueda expresarse libremente haciendo uso fluído de sus experiencias y saberes, compartiéndolo con sus pares, además de lograr que este se motive en el proceso de su aprendizaje, se estaría logrando un gran aporte al desarrollo de su creatividad, debido a que genera el hábito de recibir y dar información de forma constante, lo cual nutriría sus conocimientos a partir del desarrollo de su creatividad narrativa que como ya se mencionó anteriormente, tiene un gran impacto en el desempeño y desenvolvimiento sobre todo del profesional psicólogo.

Es importante también mencionar que la segunda dimensión, Creatividad gráfica, posee cuatro indicadores: originalidad, elaboración, título y detalles especiales. Al indagar en ello se obtuvo que el indicador de mayor peso en la creatividad gráfica es la originalidad, pero que ésta a su vez no es significativa estadísticamente. Lo cual encuentra contradicción con Castillo (2007) en su investigación realizada "Creatividad y uso de desechos como material educativo en la educación universitaria" concluyó que desarrollar las habilidades de los estudiantes implementando actividades que generen su pensamiento creativo y utilización de recursos innovadores, definitivamente incrementará aún más sus niveles de creatividad, aspectos muy importantes que a la vez mejoran su aprendizaje. Si bien anteriormente se encontró que la creatividad narrativa tiene un mayor impacto en el quehacer profesional de los psicólogos, la creatividad gráfica no deja de ser un elemento importante a desarrollar para la mejora de los jóvenes. El indicador de originalidad puede no resultar tan determinante dentro de la creatividad gráfica, sin embargo, como lo menciona Castillo (2007), resulta importante motivar a los estudiantes a alimentar este indicador, puesto que a diario en su 
labor académica y futuro laboral se verá inmerso en una variedad de dificultades que le demandarán el uso de todas sus capacidades y dentro de ello, la originalidad contenida en la creatividad gráfica, pues se recordará a Artola et al. (2008, p. 14) que mencionaron que la Imaginación creativa guarda una gran relación con las imágenes mentales, con el visualizar y construir a partir de ello soluciones a los problemas o dificultades.

\section{Referencias}

Arango, V. y Henao, C. (2006). La imaginación creativa en la narrativa y el dibujo. Revista electrónica de Educación y Psicología, 2(3), 1-19. Recuperado de http://revistas.utp.edu.co/index.php/repes/article/view/5265/2529

Artola, T., Ancillo, I., Barraca, J. y Mosteiro, P. (2008). Manual de La prueba de imaginación creativa. Madrid: TEA Ediciones.

Barceló, J. (1989). Utopía y Racionalismo. Ciencia Política No. 17. IV Trimestre. Págs. $115-121$.

Cardona, C. (2016). El desarrollo de la creatividad y su relación con el rendimiento académico en las áreas de lenguaje, matemática y artística en estudiantes universitarios (Trabajo de fin de Grado). Universidad Internacional de La Rioja, España. Recuperado de https://reunir.unir.net/bitstream/handle/123456789/4550/CARDONA\%20REYES\% 2c\%20CLAUDIA\%20XIMENA.pdf?sequence $=1 \&$ is Allowed $=\mathrm{y}$

Casillas, M. (1999). Aspectos importantes de la creatividad para trabajar en el aula. Revista digital de educación "Nueva época", 10, 1-8. Recuperado de http://www.jmunozy.org/files/NEE/sobredotado/Materiales_poz/7.materiales/creativ idad/creatividad_en_el_aula.pdf

Castillo, D. (2007). Creatividad y uso de desechos como material educativo en la educación universitaria (Tesis doctoral). Universidad Nacional Mayor de San Marcos, Lima, Perú. Recuperado de http://cybertesis.unmsm.edu.pe/bitstream/handle/cybertesis/2432/Castillo_bd.pdf?se quence $=1 \&$ is Allowed $=\mathrm{y}$

Esquivias, M. (2004). Definiciones, antecedentes y aportaciones. Revista Digital Universitaria, 5(1), 1-17. Recuperado de http://www.revista.unam.mx/vol.5/num1/art4/ene_art4.pdf

Florida, R., Mellander, C. y King, K. (2015). The global creativity index 2015. Martin Prosperity Institute. University of Toronto. Estados Unidos. 
González, C. (1998). Arte, arte literario y concreción estética. Contextos, Estudios de Humanidades y Ciencias Sociales, 1, 131-134. Recuperado de http://revistas.umce.cl/index.php/contextos/article/view/925/906

Hernández, R., Fernandez C., Baptista, L. (2014). Metodología de la investigación sexta edición. D. F. México. McGraw-Hill Interamericana.

Herrera, H. (2015). La imaginación en la semiosis peirceana como componente creativo del signo. En (APELLIDO DEL PRESIDENTE), Congreso Latinoamericano de Teoría Social. Congreso llevado a cabo en el Congreso Latinoamericano de Teoría Social. Instituto de Investigaciones Gino Germani. Facultad de Ciencias Sociales, Universidad de Buenos Aires, Buenos Aires, Argentina. Recuperado de: http://cdsa.aacademica.org/000-079/74.pdf

Mas, C. y Medinas, M. (2007). Motivaciones para el estudio en universitarios. Anales de Psicología, 23(1), 17-24. Recuperado de http://www.redalyc.org/articulo.oa?id=16723103

Polanco, A. (2005). La motivación en los estudiantes universitarios. Actualidades investigativas en educación, 5(2), 1-13. Recuperado de http://www.redalyc.org/articulo.oa?id=44750219

Reyes, L. (2013). El Debate dramatizado y el desarrollo de capacidades creativas de estudiantes en el curso taller de comunicación integral en la Facultad de Educación de la Universidad Nacional Mayor de San Marcos (Tesis doctoral). Universidad Nacional Mayor de San Marcos, Lima, Perú. Recuperado de http://cybertesis.unmsm.edu.pe/handle/cybertesis/3075

Ruschiensky, C. (2015). Competence and Creativity in Translation: Multilingual Perspectives (Tesis doctoral). Concordia University Montreal, Quebec, Canada. Recuperado https://spectrum.library.concordia.ca/979835/1/Ruschiensky MA S2015.pdf

Serna, A. (2016). Creatividad, inteligencias múltiples y estrategias de aprendizaje en maestras y maestros en formación (Trabajo Fin de Máster). Universidad Internacional de la Rioja, España. Recuperado de https://reunir.unir.net/bitstream/handle/123456789/4553/SERNA\%20JARAMILLO \%2c\%20ADRIANA\%20JULIET.pdf?sequence $=1 \&$ is Allowed $=\mathrm{y}$

Sternberg, R. J. (1999). Estilos de pensamiento. Claves para identificar nuestro modo de pensar y enriquecer nuestra capacidad de reflexión. Paidós, Barcelona.

Sternberg, R., Lubart, T. (1997) La creatividad en una cultura conformista. Un desafío a las masas. Barcelona: Paidós.

UNESCO. (2008). Artes y creatividad. Crear en el plano nacional e internacional un marco jurídico propicio al desarrollo de la creatividad y la práctica de las artes. 


\begin{abstract}
Recuperado
de

http://portal.unesco.org/culture/es/ev.php-

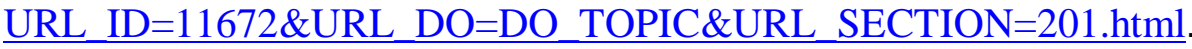

